(pp 447-454)

\title{
Quantitative Analysis and Recovery Process of a Patient with Ataxic Upper Limb Using a Robotic Device
}

Toshiyuki AOYAMA, PT, MSc

Department of Physical Therapy, Ibaraki Prefectural University of Health Sciences

Fuminari KANEKO, PT, PhD, Nobuhiro AOKI, PT, MSc

Second Division of Physical Therapy, School of Health Science, Sapporo Medical University

Tatsunori SAWADA, OT, PhD

Division of Rehabilitation, IMS Itabashi Rehabilitation Hospital

Tatsuya HAYAMI, PhD

Division of Health Science Education, School of General Education Shinshu University

Purpose: The purpose of the present study is to investigate the kinematic change in a patient with upper limb ataxia, quantitatively using a robotic device.

Methods: One septuagenarian man with upper limb ataxia due to midbrain infarction participated in this study. The kinematic assessment was performed at baseline (BL) and 2 and 4 weeks after baseline $(2 \mathrm{~W}, 4 \mathrm{~W})$ during his rehabilitation at the hospital. The subject made use of visually guided tracking movement in the horizontal plane with his affected arm using a robotic device. The six kinematic parameters (coordinate error, velocity error, moving range of the $\mathrm{X}$-axis and Y-axis, total length of trajectory, and Jerk Index) were calculated. Clinical findings such as the finger-nose test and the hand pronation supination test were also obtained.

Results: Coordinate error gradually decreased, but the other 5 parameters increased at $2 \mathrm{~W}$ and decreased at $4 \mathrm{~W}$. The finger-nose test and the hand pronation supination test were positive at both $\mathrm{BL}$ and $4 \mathrm{~W}$.

Conclusion: The kinematic change of the upper limb tracking movement may not simply reflect the change of ataxic symptom but also an adaptive motor strategy. The present results indicate that the kinematic assessment using this robotic device is useful for detecting chronological change of the ataxic arm movement quantitatively. 